\title{
Proceso de Industrialización a nivel de Planta Piloto de la Oca (Oxalis Tuberosa)
}

Recepción: Enero de 2008 / Aceptación: Mayo de 2008

(1)Julio Antolin Yenque Dedios

${ }^{(2)}$ Mooner Aurelio Lavado Soto ${ }^{(3)}$ Eulogio Guillermo Santos de la Cruz

\begin{abstract}
RESUMEN
El presente trabajo de investigación trata del diseño experimental de un proceso para su aplicación a nivel de planta piloto en la industrialización de la oca, orientado a la elaboración de néctar mix (mezcla de oca con tuna) y confitado de oca. En los experimentos de elaboración del néctar se ha determinado que la mezcla de mejores características organolépticas es la que se formula con un $20 \%$ de tuna. En lo que respecta al confitado, se utilizó jarabe de sacarosa de 30 a $75^{\circ}$ Brix de concentración, hallándose que el tiempo óptimo de cada inmersión es de 24 horas.
\end{abstract}

Palabras Clave: Oca, Confitado y Néctar.

INDUSTRIALIZATION PROCESS LEVEL OF OCA PILOT PLANT (OCA)

\section{ABSTRACT}

This research work is the experimental design of a process for application-level pilot plant in the industrialization of the goose, aimed at developing nectar mix (mixture of goose with tuna) and confit of goose. In experiments development of nectar it has been determined that the mixture of improved organoleptic characteristics in which it made with a $20 \%$ Pear. Regarding the confit was used sucrose syrup from 30 to $75^{\circ}$ Brix concentration being found that the optimum time for each dive is 24 hours.

Keywords: Oca, Confitado and Nectar.

\section{INTRODUCCIÓN}

La oca (oxalis tuberosa), es un tubérculo que se cultiva entre $3200-$ 3900 m.s.n.m. Contiene proteínas, carbohidratos y vitamina C (ácido ascórbico). A fin de darle un valor agregado y analizar la posibilidad de su industrialización, se diseña un procedimiento experimental de aplicación a nivel de planta piloto, para la elaboración de néctar mix (mezcla de oca con $20 \%$ de tuna) y confitado como sucedáneo de fruta confitada, que constituye el objetivo del presente estudio de investigación.

El néctar mix es un producto constituido por una mezcla de pulpa de oca y tuna, azúcar, ácido cítrico, perseverante químico, estabilizador y agua.

El confitado de la oca es un producto constituido por cubos de $5-8 \mathrm{~mm}$ del tubérculo, sometido a inmersión en un jarabe de azúcar de concentraciones que varían entre $30-75^{\circ}$ Brix, durante 24 horas de duración cada una.

La oferta de la materia prima (Oca) que permita proyectar la demanda del insumo para su posible industrialización a nivel nacional, se ilustra en el gráfico $\mathrm{N}^{\circ} 1$.

\section{MATERIALES Y EQUIPO}

\section{Materia Prima}

Se trabajó con oca procedente de la comunidad de Seccha, localidad anexa a La Unión, capital de la provincia de Dos de Mayo (Huánuco). La tuna se adquirió en el mercado MINKA.

\section{Soleado del tubérculo}

Los carbohidratos de la oca, al ser expuestos al sol se transforman en azúcar. La evolución del contenido de este último con el tiempo de exposición al sol, se aprecia en el cuadro $N^{\circ} 1$. El incremento del contenido de azúcar con el tiempo de soleado, permitirá un menor consumo de azúcar corriente (sacarosa) en la formulación del producto.

(1) Ingeniero Industrial. Coordinador del Departamento de Diseño y Tecnología Industrial. UNMSM E-mail: jyenque@unmsm.edu.pe

(2) Magíster en Ciencias. Profesor del Departamento de Diseño y Tecnología Industrial. UNMSM E-mail: esantosd@unmsm.edu.pe

(3) Ingeniero Químico. Profesor del Departamento de Diseño y Tecnología Industrial. UNMSM E-mail: mlavados@unmsm.edu..pe 


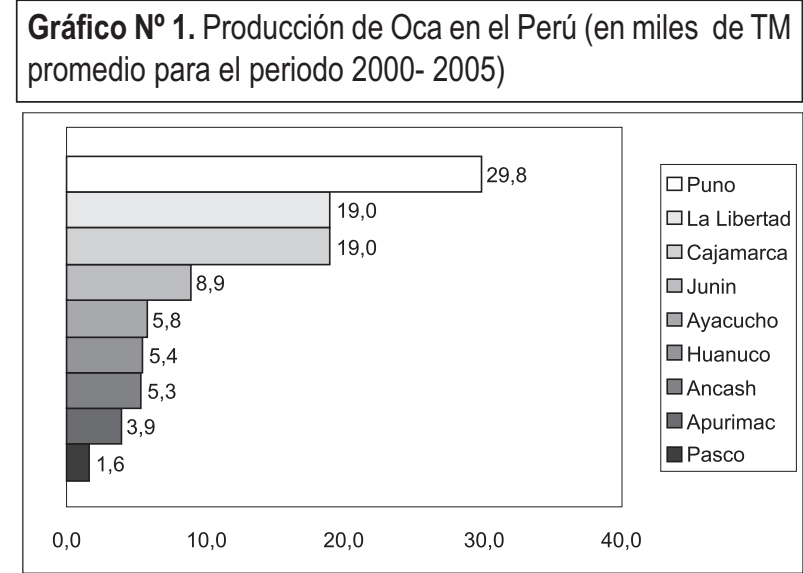

Fuente: MINAG

\begin{tabular}{|c|c|}
\hline \multicolumn{2}{|c|}{$\begin{array}{c}\text { Cuadro } \\
\mathbf{N}^{\mathbf{0}} \\
\text { contenido de azúcar }\end{array}$} \\
\hline $\begin{array}{c}\text { TIEMPO } \\
\text { (días) }\end{array}$ & $\begin{array}{c}\text { CONTENIDO DE AZÚCAR } \\
\text { (oBrix) }\end{array}$ \\
\hline 0 & 7.5 \\
3 & 11.0 \\
5 & 12.0 \\
7 & 12.5 \\
10 & 13.5 \\
20 & 15.0 \\
\hline
\end{tabular}

Fuente: ELABORACIÓN PROPIA

Las cantidades anteriores se pueden graficar en un par de ejes rectangulares, tal como se aprecia en el grafico $\mathrm{N}^{\circ} 2$.

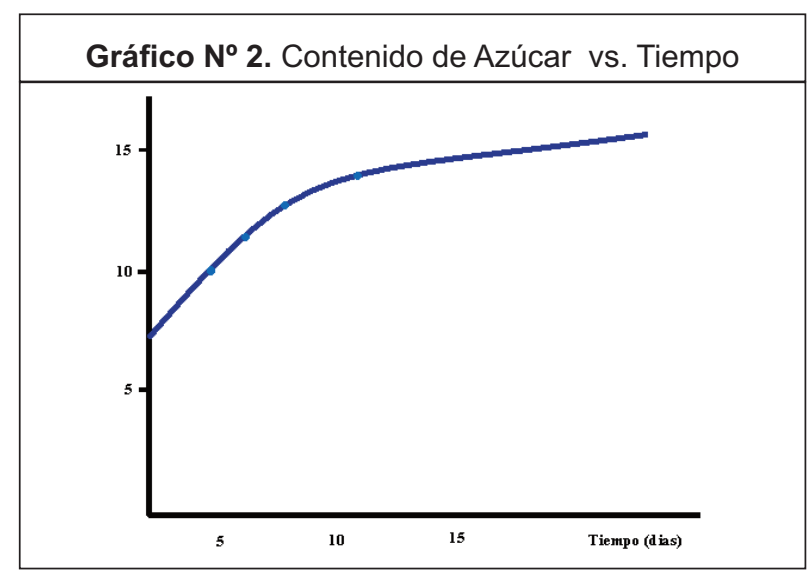

Fuente: ELABORACIÓN PROPIA

\section{Aditivos}

Los aditivos permitidos utilizados para la elaboración de los productos son:

\begin{tabular}{|c|c|}
\hline $\begin{array}{c}\text { NÉCTAR } \\
\text { CONFITADO }\end{array}$ & CMC \\
\hline $\begin{array}{l}\text { ácido cítrico } \\
\text { ácido ascórbico } \\
\text { bisulfato de sodio } \\
\text { sorbato de potasio } \\
\text { sacarosa }\end{array}$ & $\begin{array}{l}\text { ácido cítrico } \\
\text { ácido ascórbico } \\
\text { bisulfito de sodio (se usa } \\
\text { para los dos productos } \\
\text { sorbato de potasio } \\
\text { sacarosa }\end{array}$ \\
\hline
\end{tabular}

\section{Equipos}

Básicamente se debe disponer de los siguientes equipos y/o instrumentos:

- Balanza (0 - $50 \mathrm{~kg})$

- Potenciómetro

- Bandejas de acero

- Refractómetro $(0$ - - Tinas (6) $80^{\circ}$ Brix) $\quad$ Coladores (3)

- Termómetro $(0$ - Cucharones (2) $\left.150^{\circ} \mathrm{C}\right)$

- Ollas de 50 L. (2)

- Horno eléctrico (se- - Cocina Industrial cador)

- Dosificador de $10 \mathrm{~L}$.

- Selladora eléctrica - Cortadora y peladora

- Pulpeadora de 50 - Envases (botellas de $\mathrm{Kg} . / \mathrm{h}$ $300 \mathrm{~mL}$ y bolsas de

- Baldes (6) polipropileno)

\section{PROCESO EXPERIMENTAL}

Para su aplicación a nivel de planta piloto el proceso experimental diseñado, corresponde a un "batch" de 320 botellas $(300 \mathrm{ml})$, de néctar por día y $25 \mathrm{Kg}$. de confitado diario (2).

\section{PROCESO DE ELABORACIÓN DEL NÉCTAR}

El flujo de operaciones para la elaboración del néctar responde al esquema que se presenta a continuación:

Gráfico $N^{\circ}$ 3. OCA

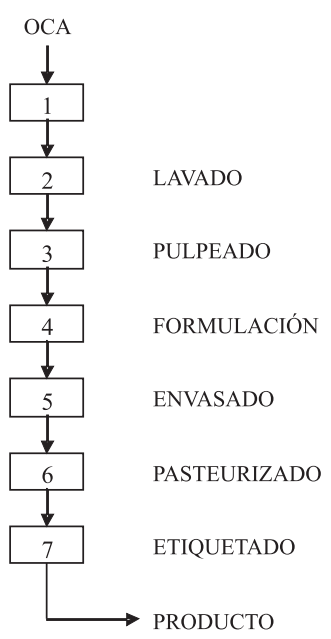

Fuente: ELABORACIÓN PROPIA 
En seguida se detallan cada uno de los pasos del proceso.

\section{Pesado}

Esta operación permite determinar el rendimiento que se expresa mediante la relación, $\left(\frac{p u l p a}{o c a}\right) \times 100 \%$. En la elaboración del néctar el rendimiento obtenido es de $90 \%$.

\section{Lavado}

Sirve para eliminar las partículas extrañas adheridas al tubérculo. A fin de eliminar microorganismos la oca se sumerge en una solución de bisulfito de sodio al $0.05 \%$

\section{Pulpeado}

Consiste en obtener la pulpa de la oca mediante la pulpeadora, luego se tamiza la pulpa pasándola por un colador de malla fina.

\section{Formulación}

\section{Esta operación involucra:}

- Dilución de la pulpa con agua (incluye la pulpa de tuna con $20 \%$ de participación).

- Regulación del pH.

- Regulación de los grados Brix (contenido de azúcar).

- Adición de estabilizador, preservante y ácido ascórbico.

- Calentamiento de la mezcla hasta llegar a la temperatura de pasteurización $\left(85^{\circ} \mathrm{C}\right.$ durante $10 \mathrm{mi}-$ nutos).

- La proporción de agua a pulpa es de 9:2 (Industrial Data, Vol 10 №1-2007).

- El pH se regula mediante la adición de ácido cítrico y/o ácido ascórbico. Por lo general, debe estar en un nivel de 3.5.

- La cantidad de azúcar ('Brix) se regula mediante la adición de azúcar blanca. Tomando en cuenta la evolución del contenido de azúcar con el tiempo de soleado del tubérculo (Cuadro $N^{\circ} 1$ ), se elabora el gráfico $\mathrm{N}^{\circ} 4$.

El contenido de estabilizador (CMC) es de $0.5 \%$ y la de preservante (sorbato de potasio), $0.1 \%$ con respecto al néctar.

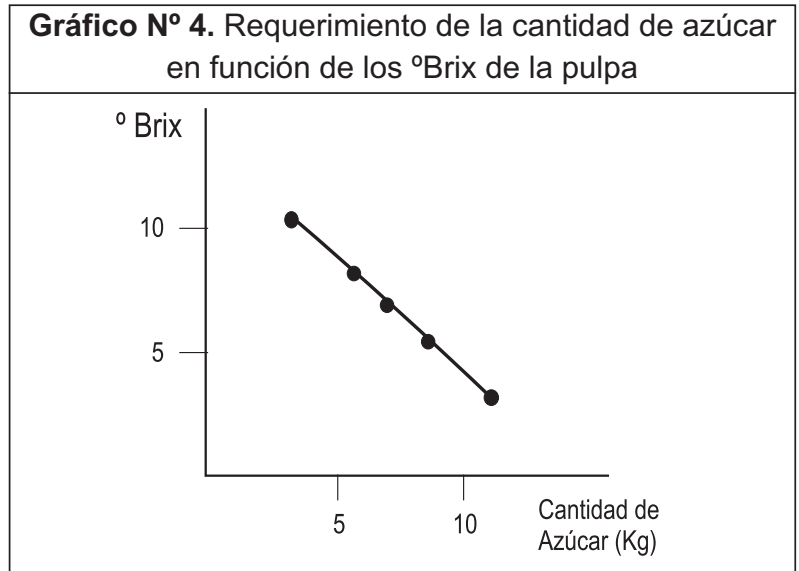

Fuente: ELABORACIÓN PROPIA

\section{Envasado}

Esta operación se realiza a la temperatura de pasteurización $\left(85^{\circ} \mathrm{C}\right)$, en botellas de vidrio de $300 \mathrm{~mL}$, cerrándolo de inmediato.

\section{Pasteurizado}

La mezcla formulada y calentada a $85^{\circ} \mathrm{C}$ durante 10 minutos se enfría rápidamente para reducir las pérdidas de aroma, sabor y consistencia. El pasteurizado tiene como función primordial la destrucción de microorganismos.

\section{Etiquetado}

Es la etapa final del proceso que se realiza cuando las botellas se encuentran totalmente enfriadas a la temperatura del medio ambiente.

\section{Proceso de elaboracion del confitado}

El flujo de operaciones para este proceso se presenta en el siguiente esquema.

OCA

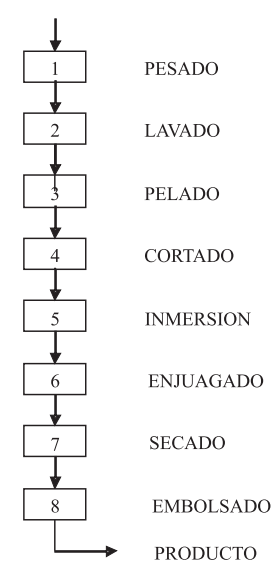

Fuente: ELABORACIÓN PROPIA 
A continuación se especifica cada una de las etapas del proceso.

\section{Pesado}

Permite determinar el rendimiento expresado mediante la relación, $\left(\frac{\text { cortes }}{\text { oca }}\right) \times 100 \%$. En la elaboración del confitado, el rendimiento obtenido es de $70 \%$.

\section{Lavado}

A fin de eliminar microorganismos la oca se lava en agua y enseguida en una solución de bisulfito de sodio al $0.05 \%$.

\section{Pelado}

El pelado se realiza en forma manual para desprender la cáscara y eliminar los extremos del tubérculo.

\section{Cortado}

La presentación de productos similares (fruta confitada) en el mercado en forma de cubos, amerita realizar cortes del tubérculo pelado en cubitos de 5-8 $\mathrm{mm}$ de arista.

\section{Inmersión}

Se realiza utilizando jarabe de azúcar de 30$75^{\circ}$ Brix. El número de inmersiones realizados es de seis, de 24 horas de duración cada una. El incremento de masa de los cubitos de oca durante la inmersión evolución según se muestra en el gráfico $\mathrm{N}^{\circ} 6$.

Gráfico $\mathbf{N}^{\circ}$ 6. Evolución del incremento de masa total de los cubitos de oca en función de los ${ }^{\circ}$ Brix del jarabe

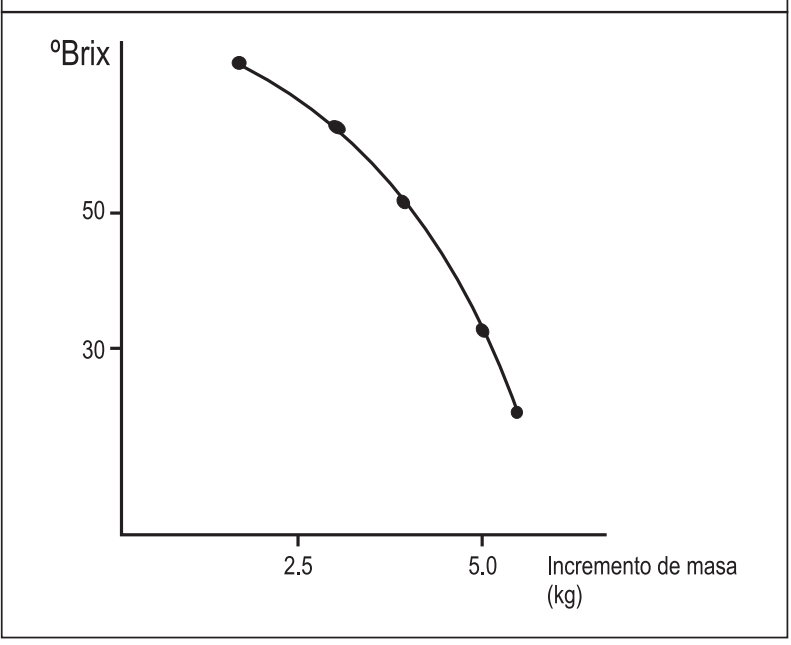

Fuente: ELABORACION PROPIA

\section{Enjuagado}

Al término de la última inmersión los cubitos se enjuagan con agua durante 30 segundos, a fin de desprender el jarabe no absorbido y que se encuentran en la superficie del confitado.

\section{Secado}

Se realiza a $70^{\circ} \mathrm{C}$ durante 20 horas. Se utilizan bandejas de acero inoxidable.

\section{Embolsado}

El producto envasa en bolsas de polipropileno de 12 $\mathrm{cm}$. x $15 \mathrm{~cm}$., Finalmente las bolsas se sellan con la selladora eléctrica.

\section{ANÁLISIS DE RESULTADOS}

En primer lugar es conveniente hacer un comentario acerca de la evolución del contenido de azúcar del tubérculo, en función del tiempo de soleado. El análisis del gráfico $\mathrm{N}^{\circ} 2$ permite apreciar que un soleado de 10 días constituye un periodo de estabilización del contenido de azúcar de la oca, alcanzado una concentración de $13.5^{\circ}$ Brix.

\section{VARIACIÓN DE LA CANTIDAD DE SACAROSA REQUERIDA}

Para determinar la cantidad de sacarosa añadida en la operación de formulación de néctar, se realiza un balance sólidos, utilizando la siguiente correlación (3).

$\left(m_{p}+m_{o}\right)\left({ }^{\circ}\right.$ Brix pulpa $)+100 m_{s}=\left(m_{s}+m_{p}+m_{a}\right)$ ( ${ }^{\circ}$ Brix nectar), donde:

$\mathrm{m}_{\mathrm{p}}=$ masa pulpa $=21 \mathrm{~kg}$.

$\mathrm{m}_{\mathrm{O}}=$ masa de oca $=46 \mathrm{~kg}$.

${ }^{\circ}$ Brix pulpa $=7.5$ a 13.5

$\mathrm{m}_{\mathrm{s}}=$ masa de sacarosa (cantidad a calcular)

$\mathrm{m}_{\mathrm{a}}=$ masa de agua $=94.5 \mathrm{~kg}$.

${ }^{\circ}$ Brix néctar $=13$

Para un ${ }^{\circ}$ Brix de 7.5 de pulpa, el resultado obtenido para la cantidad de sacarosa es de $11.5 \mathrm{~kg}$. Los cálculos realizados utilizando los diferentes ${ }^{\circ} \mathrm{Brix}$ de la pulpa se muestran en el gráfico $\mathrm{N}^{\circ} 4$. El análisis del mismo permite establecer que el soleado prolongado del tubérculo, representa un menor consumo de azúcar blanca (sacarosa), con una variación de $4.6 \mathrm{~kg}$. entre la oca sin solear y la soleada durante 10 días. 


\section{INCREMENTO DE LA MASA DE LOS CUBITOS POR ABSORCIÓN DE JARABE}

En el gráfico $N^{\circ} 6$ se observa la evolución del incremento de la masa de los cubitos durante la operación de inmersión de los mismos en el jarabe de azúcar. La mayor saturación se produce cuando el jarabe tiene baja concentración (30 $\left.{ }^{\circ} \mathrm{Brix}\right)$, alcanzando una menor absorción de jarabe o saturación, para una concentración de $75^{\circ}$ Brix, constituyendo este un índice de finalización de las etapas de inmersión.

\section{CONCLUSIONES}

El tiempo óptimo de soleado del tubérculo es de 10 días, período durante el cual el contenido de azúcar del mismo, tiende a estabilizarse en $13.5^{\circ}$ Brix.

Para un néctar de $13^{\circ}$ Brix, como el que se ha obtenido, la cantidad de azúcar (sacarosa) añadida a la mezcla de los diferentes insumos (formulación), dis- minuye un $40 \%$ tomando como base de cálculos los extremos de los grados Brix del tubérculo.

Las diferentes concentraciones del jarabe para la inmersión de los cubitos de oca, tienden a una mínima saturación o absorción de la solución alrededor de $75^{\circ}$ Brix de concentración del mismo.

La regulación del pH del néctar mediante inclusión del ácido ascórbico permite mantener el contenido de vitamina $C$ en el nivel inicial del tubérculo (38mg/100g. de oca)

\section{REFERENCIAS BIBLIOGRÁFICAS}

1. Industrial Data Vol.10, No. 1 - 2007, Págs. 7 - 10

2. Madrid Vicente, Madrid Cenzano, Nuevo Manual de Industrias Alimentarías. AMV EDICIONES, MUNDI - PRENSA, 2001.

3. ITDG - PERU, UNIFEM, Procesamiento de tubérculos, 1998. 